\title{
Calculation of Transient Motion of Submerged Cables
}

\section{By Thomas S. Walton and Harry Polachek}

\begin{abstract}
The system of nonlinear partial differential equations governing the transient motion of a cable immersed in a fluid is solved by finite difference methods. This problem may be considered a generalization of the classical vibrating string problem in the following respects: a) the motion is two dimensional, b; large displacements are permitted, c) forces due to the weight of the cable, buoyancy, drag and virtual inertia of the medium are included, and d) the properties of the cable need not be uniform. The numerical solution of this system of equations presents a number of interesting mathematical problems related to: a) the nonlinear nature of the equations, b) the determination of a stable numerical procedure, and c) the determination of an effective computational method. The solution of this problem is of practical significance in the calculation of the transient forces acting on mooring and towing lines which are subjected to arbitrarily prescribed motions.
\end{abstract}

1. Introduction. This problem arose as a result of an urgent requirement by the Navy in connection with a series of nuclear explosion tests which were conducted in the Pacific. In preparation for these tests a number of ships were instrumented and moored at specified locations from the explosion point. These positions had to be maintained intact during the period preceding the explosion. However, the bobbing up and down of the ships due to ocean waves could excite transient forces in the mooring lines sufficient to break them and thus result in the loss of information from the tests. Several months prior to these tests a request was made to the Applied Mathematics Laboratory to calculate the magnitude of the forces acting on the mooring lines for waves of varying amplitude and frequency. The two factors which made a theoretical solution feasible at this time, whereas it would not have been possible several years ago, were: a) the availability of a high-speed computer and b) the recent progress made in the understanding and development of numerical methods for the solution of systems of partial differential equations by finite-difference methods.

Although this problem was solved to satisfy a specific request, it is more useful to regard it as the general problem of the two-dimensional motion of a cable or rope immersed in a fluid, and it becomes immediately apparent that its solution is applicable to a wide class of engineering problems involving the motion of cables. such as: a) the laying of submarine telegraph cables, b) the towing of a ship or other object in water, or c) the snapping of power lines as a result of transient forces caused by storms. The problem may be stated abstractly as follows: Given the initial conditions (i.e., position and velocity at any time, $t_{0}$ ) and boundary conditions (positions of end points at all times) of a cable immersed in a fluid, determine its subsequent motions. The motions are assumed to take place in two dimensions.

Received September 24, 1959. This work was done at the Applied Mathematics Laboratory, David Taylor Model Basin, and the paper is a condensation of DTMB Report 1279 [1]. 
Forces that are assumed acting on the cable are: a) forced motion of the extremities of the cable, b) damping or drag as it moves through the fluid, c) inertial reaction of the surrounding fluid, d) weight of the cable, and e) buoyancy. Variations in the mass as well as other physical properties of the cable along its length are allowed. However, in the present solution it is assumed that the cable is inextensible. The displacements may be large and the motions rapid, provided that all significant components of the driving motion lie in a frequency range well below the lowest natural frequency of the line for elastic (longitudinal) vibrations. In other words, the cable must be sufficiently short (or the velocity of propagation of elastic waves sufficiently great) that the line may be considered to be in equilibrium as far as longitudinal waves are concerned. In subsequent work the authors have carried out solutions for cables with elastic properties.

2. Derivation of Equations of Motion. The problem under consideration is a generalization of the classical problem of the motion of a vibrating string. We wish to deduce the approximate motion of a flexible steel cable without becoming involved in the explicit computation of the elastic forces which act on the cable. However, the formulation of the problem will be more general in several respects, namely:

a) Longitudinal as well as transverse motions of the line must be taken into consideration.

b) The occurrence of large displacements from the equilibrium configuration of the line must be permitted.

c) The extremities of the cable may be at different levels with the cable sagging between the positions of support. 'This requires that the weight of the line be taken into account. Thus, even when the line is in statis, equilibrium, the tension will not be uniform nor will the line be straight.

d) Since the cable is submerged, the static forces must include the buoyancy of the medium, and the dynamic forces must allow for the virtual inertia of the medium. Furthermore, it is necessary to make provision for damping forces due to the drag on the line whenever transverse motion is occurring.

e) Finally, it is desired to suspend concentrated loads at one or more points along the line and to change the diameter and linear density of the cable at specified points.

The best approach to the solution of a problem with such general specifications appears to be $a$-numerical method based on finite-difference approximations. Inasmuch as we are committing ourselves to the eventual use of differences in both the time and space dimensions, it will be simpler to introduce the spacewise discreteness into the original formulation of the problem. We therefore proceed at once to the derivation of the equations of motion of a simplified model in which the distributed mass of the cable has been replaced by a series of discrete masses $\boldsymbol{m}_{j}$ attached to a weightless, inextensible line. This leads to a system of ordinary differential equations. It may be shown that, in the limit, the resulting equations pass over into the corresponding partial differential equations for the motion of a submerged cable.

Figure 1 shows a typical configuration of the system with the cable attached to a float at the surface and anchored to the bottom. Also, a heavy load is suspended 


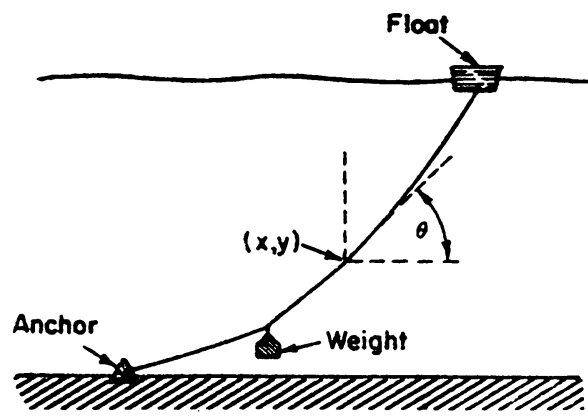

Frg. 1. -Mooring line.

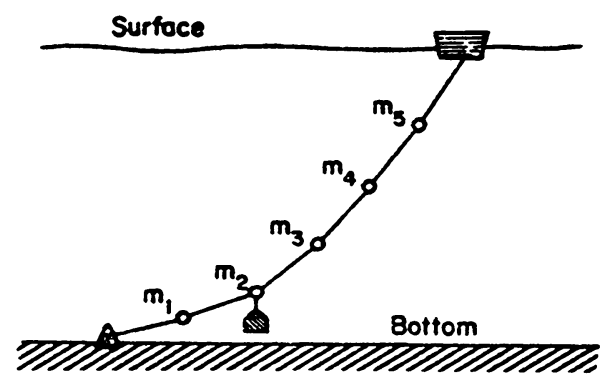

FIG. 2. -Discrete representation.

from a point near one end of the cable. Other boundary conditions are possible, but the equations of motion will be the same in any case. The horizontal and vertical coordinates of a point on the line are called $x$ and $y$, respectively, and the angle between the horizontal and the tangent to the line is designated by $\theta$. Figure 2 illustrates the corresponding discrete model for which the equations will actually be derived. The line is divided into segments in such a way that there will always be an integral number of them between any points where an abrupt change in some parameter occurs. The junctions between the segments are numbered according to the subscript index $j$, which runs from 0 at the anchor to $s$ at the surface.

Before we can properly invoke Newton's law of motion, it is necessary to consider the inertial properties of the fluid in which the cable is immersed. We shall assume that the kinetic energy imparted to the surrounding medium is independent of the component of velocity parallel to the line, whereas it varies as the square of the component of velocity at right angles to the line. Thus, when an element of the cable is accelerated longitudinally, no hydrodynamic reaction occurs, but when the cable is accelerated transversely, it behaves as though it possessed additional inertia. For convenience in formulating the equations of motion in Cartesian coordinates, the transverse component of acceleration, namely, $-\ddot{x} \sin \theta+\ddot{y} \cos \theta$, can be further resolved into horizontal and vertical components (to which the corresponding components of the accompanying inertial reaction will be proportional). That is,

$$
\begin{aligned}
& \text { Horizontal component }=\ddot{x} \sin ^{2} \theta-\ddot{y} \sin \theta \cos \theta \\
& \text { Vertical component }=\ddot{y} \cos ^{2} \theta-\ddot{x} \sin \theta \cos \theta
\end{aligned}
$$

Thus, each component of the hydrodynamic reaction depends on both components of acceleration. In general, the reaction force is not parallel to the acceleration vector (except when the tangential component is zero), so that it is necessary to regard the inertial parameters of the system as tensors rather than simple scalars.

The differential equations governing the motion of the $j$ th station on the line (see Fig. 2) can be written in matrix notation as follows:

$$
\left(\begin{array}{cc}
I_{j} & -K_{j} \\
-K_{j} & J_{j}
\end{array}\right)\left(\begin{array}{l}
\ddot{x}_{j} \\
\ddot{y}_{j}
\end{array}\right)=\left(\begin{array}{c}
F_{j}{ }^{X} \\
F_{j}{ }^{Y}
\end{array}\right)
$$


where

$$
\begin{aligned}
& I_{j}=m_{j}+\frac{1}{2}\left(e_{j+1} \sin ^{2} \theta_{j+1}+e_{j-1} \sin ^{2} \theta_{j-1}\right)+m_{j}^{x} \\
& J_{j}=m_{j}+\frac{1}{2}\left(e_{j+1} \cos ^{2} \theta_{j+1}+e_{j-1} \cos ^{2} \theta_{j-1}\right)+m_{j}{ }^{2} \\
& K_{j}=\frac{1}{2}\left(e_{j+1} \sin \theta_{j+1} \cos \theta_{j+1}+e_{j-1} \sin \theta_{j-1} \cos \theta_{j-1}\right)
\end{aligned}
$$

and $\dagger$

$$
\begin{array}{ll}
m_{j} & =\frac{1}{2}\left[\mu_{j+1} l_{j+1}+\mu_{j-1} l_{j-1}\right] \\
e_{j+1} & =\rho k_{j+1} l_{j+1} \sigma_{j+1} \\
m_{j}^{x} & =m_{j}^{*}+\rho V_{j}^{X} \\
m_{j} & =m_{j}^{*}+\rho V_{j}^{Y} \\
\cos \theta_{j+1} & =\left(x_{j+1}-x_{j}\right) / l_{j+1} \\
\sin \theta_{j+1} & =\left(y_{j+1}-y_{j}\right) / l_{j+1} .
\end{array}
$$

Each lumped mass, $m_{j}$, has been expressed as the average mass of the two segments of cable which lic on either side of station $j$. Also, one-half the equivalent transverse mass, $e_{j}$, of the fluid entrained with each of these segments has been included in the inertia tensor. Furthermore, at those stations from which a weight is suspended, the effective horizontal and vertical masses, $m_{j}{ }^{x}$ and $m_{j}{ }^{x}$, of the weight are to be added. For simplicity in allowing for virtual inertia, we have assumed that any such weights possess a certain degree of symmetry and remain upright as the line moves about.

The force vector, $F_{j}$, on the right side of eq. (2.1) can be expressed as the sum of internal forces (the tensions acting between adjacent mass elements) and whatever external forces are present. Thus; in expanded form the equations of motion can be written

$$
\begin{aligned}
I_{j} \ddot{x}_{j}-K_{j} \ddot{y}_{j} & =T_{j+\frac{1}{1}} \cos \theta_{j+\frac{1}{2}}-T_{j-1} \cos \theta_{j-1}+X_{j} \\
-K_{j} \ddot{x}_{j}+J_{j} \ddot{y}_{j} & =T_{j+\frac{1}{1}} \sin \theta_{j+\frac{1}{1}}-T_{j-1} \sin \theta_{j-1}+Y_{j}
\end{aligned}
$$

where $T_{j+1}=$ tension in segment of line between stations $j$ and $j+1$

$X_{j}=$ horizontal component of resultant external force at station $j$

$Y_{j}=$ vertical component of resultant external force at station $j$.

There are two sources of external force, namely: 1) gravity, which gives rise to the weight minus the buoyancy and acts only in the vertical, and 2) fluid resistance, which gives rise to the damping forces. Thus, we write

$$
\begin{aligned}
& X_{j}=-\frac{1}{2}\left[D_{j+\frac{1}{1}} \sin \theta_{j+1}+D_{j-1} \sin \theta_{j-\frac{1}{1}}\right]+X_{j}^{*} \\
& Y_{j}=\frac{1}{2}\left[D_{j+\frac{1}{3}} \cos \theta_{j+1}+D_{j-\frac{1}{1}} \cos \theta_{j-1}\right]+Y_{j}^{*}-W_{j}-W_{j}^{*}
\end{aligned}
$$

where

$$
\begin{aligned}
& W_{j}=m_{j} g-\frac{1}{2} \rho g\left(l_{j+\xi} \sigma_{j+\frac{1}{j}}+l_{\left.j-\frac{j}{j} \sigma_{j-1}\right)}\right. \\
& W_{j}^{*}=m_{j}^{*} g-\rho g V i^{*}
\end{aligned}
$$

† See Appendix for definitions of the pas ameters which appear in the formulas. 
and $D_{j+1}=$ drag on segment of line between stations $j$ and $j+1$

$X_{j}^{*}=$ horizontal component of damping force on weight at station $j$

$Y_{j}^{*}=$ vertical component of damping force on weight at station $j$.

Again, in order to get the best approximation to the continuous case, the net effect of the drag at station $j$ has been expressed as one-half of each component of the drag on the segments which lie on either side of this station. The buoyant force of the displaced fluid has been treated likewise.

We have assumed that the drag, $D_{j+\frac{1}{3}}$, on a segment of the line acts in a direction at right angles to the line. This is a good approximation whenever the velocity is high enough to produce significant forces, since at all but the lowest Reynolds numbers the tangential component of the hydrodynamic force is very small compared to the normal component. Furthermore, we assume that the drag is proportional to the square of the component of relative velocity normal to the line:

$$
D_{j+1}=-f_{j+1}^{D} q_{j+1}\left|q_{j+1}\right|
$$

where

$$
\begin{aligned}
f_{j+1}^{D} & =\frac{1}{2} \rho C_{j+\frac{1}{3}}^{D} l_{j+\frac{1}{3}} d_{j+1} \\
q_{j+\frac{1}{2}} & =-\frac{1}{2}\left[\left(\dot{x}_{j+1}-c\right)+\left(\dot{x}_{j}-c\right)\right] \sin \theta_{j+\frac{1}{3}}+\frac{1}{2}\left[\dot{y}_{j+1}+\dot{y}_{j}\right] \cos \theta_{j+\frac{1}{1}} .
\end{aligned}
$$

The positive normal to the line has been arbitrarily taken to be directed upward when $\theta$ equals zero. The use of the minus sign and the introduction of the absolute value of one of the velocity factors ensures that the drag will always be opposed to the direction of $q_{j+1}$ and thus act as a dissipative force to remove energy from the system. Since the velocities of the two endpoints of each segment will, in general, differ slightly, their mean value (which for a straight line segment is exactly equal to the velocity of the midpoint) is taken as a representative value in the definition of $q_{j+1}$. In addition, the definition allows for the presence of a uniform horizontal current, $c$, to incorporate the ability to treat towing lines as well as mooring lines (or mooring lines subjected to ocean currents).

In addition to the drag on the line itself, there will also be resistance to the motion of any concentrated loads which may be suspended from the line. These additional damping forces will vary with the velocity but will not, in generäl, be directed exactly opposite to the motion of each weight. However, on account of the assumed orientation and symmetry of any such weights, the resistance force will be parallel to the velocity vector whenever the relative motion is either purely horizontal or purely vertical. Accordingly, the two components of resistance may be written

$$
\begin{aligned}
& X_{j}^{*}=-f_{j}{ }^{\boldsymbol{x}} u_{j}\left(\dot{x}_{j}-c\right) \\
& Y_{j}{ }^{*}=-f_{j}{ }^{\boldsymbol{Y}} u_{j} \dot{y}_{j}
\end{aligned}
$$

where

$$
\begin{aligned}
f_{j}^{X} & =\frac{1}{2} \rho C_{j}{ }^{X} S_{j}{ }^{X} \\
f_{j}{ }^{X} & =\frac{1}{2} \rho C_{j}{ }^{X} S_{j}{ }^{X} \\
u_{j} & =\left[\left(\dot{x}_{j}-c\right)^{2}+\dot{y}_{j}{ }^{2}\right]^{z}
\end{aligned}
$$


Up to this point an explicit formula has been given for the evaluation of every term in the equations of motion (2.2) with the exception of the tensions. To determine these we must invoke the inextensibility condition which was assumed at the outset. This takes the form of a constraint on the motion of the line. It requires that the separation between adjacent stations must not change with time. Thus, we write ${ }^{\cdot}$

$$
\left(x_{j}-x_{j-1}\right)^{2}+\left(y_{j}-y_{j-1}\right)^{2}=l_{j-1}^{2}=\text { const. }
$$

This holds for each segment of the line, and we require that the corresponding set of tensions, $T_{j-1}$, take on values such that the resulting solution of the equations of motion will be consistent with eq. (2.6). Because of the implicit nature of this condition, we are led to a system of algebraic equations for the determination of the proper tensions. At the extremities of the line $(j=0$ and $j=s) x_{j}$ and $y_{j}$ must be obtained from the boundary conditions, namely:

$$
\begin{array}{ll}
x_{0}=x_{0}(t), & y_{0}=y_{0}(t) \\
x_{*}=x_{s}(t), & y_{s}=y_{s}(t) .
\end{array}
$$

These are given as functions of time, and permit the introduction of any desired types of driving motions.

Finally, to complete the formulation of the problem a set of initial conditions must be given for each station on the line. Since the equations of motion are of the second order, it is nec:essary to specify both the coordinates and the velocities at $t=0$. That is,

$$
\begin{array}{lll}
x_{j}(0)=x_{j}{ }^{0}, & y_{j}(0)=y_{j}^{0} & (j=1,2, \cdots s-1) \\
\dot{x}_{j}(0)=\dot{x}_{j}{ }^{0}, & \dot{y}_{j}(0)=\dot{y}_{j}^{0} & (j=1,2, \cdots s-1)
\end{array}
$$

where the superscript index " 0 " is used to designate a value at the origin in time.

\section{Solution of Equations by Finite Differences.}

A. General Description of Computational Procedure. The equations governing the motion of a cable, as derived in the last section, are summarized here. The basic equations of motion, (2.2), are repeated for convenience,

$$
\begin{aligned}
I_{j} \ddot{x}_{j}-K_{j} \ddot{y}_{j}=T_{j+\frac{1}{3}} \cos \theta_{j+1}-T_{j-1} \cos \theta_{j-1}+ & X_{j} \\
& (j=1,2, \cdots s-1), \\
-K_{j} \ddot{x}_{j}+J_{j} \ddot{y}_{j}=T_{j+\frac{1}{3}} \sin \theta_{j+\frac{1}{3}}-T_{j \rightarrow \frac{1}{3}} \sin \theta_{j \rightarrow}+ & Y_{j} \\
& (j=1,2, \cdots s-1),
\end{aligned}
$$

where

a) $s$ is the number of segments into which the cable is divided,

b) $I_{j}, K_{j}, J_{j}$ are given in eq. (2.1) and are functions of the physical properties of the cable and of position only,

c) $X_{j}, Y_{j}$ are given by eqs. (2.3), (2.4), and (2.5) and are functions of the physical properties of the cable and of position and velocity.

In addition, the motion is governed by the condition of inextensibility of the cable, 
eq. (2.6), namely,

$$
\left(x_{j}-x_{j-1}\right)^{2}+\left(y_{j}-y_{j-1}\right)^{2}=l_{j-\frac{1}{2}}^{2}=\text { consu. }
$$$$
(j=1,2, \cdots s) \text {. }
$$

The differentiated (with respect to time) forms of this relation,

$$
\begin{gathered}
\left(x_{j}-x_{j-1}\right)\left(\dot{x}_{j}-\dot{x}_{j-1}\right)+\left(y_{j}-y_{j-1}\right)\left(\dot{y}_{j}-\dot{y}_{j-1}\right)=0 \\
\left(x_{j}-x_{j-1}\right)\left(\ddot{x}_{j}-\ddot{x}_{j-1}\right)+\left(y_{j}-y_{j-1}\right)\left(\ddot{y}_{j}-\ddot{y}_{j-1}\right) \\
+\left(\dot{x}_{j}-\dot{x}_{j-1}\right)^{2}+\left(\dot{y}_{j}-\dot{y}_{j-1}\right)^{2}=0 \quad(j=1,2, \cdots s),
\end{gathered}
$$

are also used in the computation.

For numerical solution by finite-difference methods the following finite-difference equivalents are used,

$$
\begin{gathered}
\dot{x}_{j}^{n+1}=\frac{x_{j}^{n+1}-x_{j}{ }^{n}}{\Delta t}, \quad \dot{y}_{j}^{n+1}=\frac{y_{j}^{n+1}-y_{j}^{n}}{\Delta t} \quad(j=1,2, \cdots s-1), \\
\ddot{x}_{j}{ }^{n}=\frac{x_{j}^{n+1}-2 x_{j}{ }^{n}+x_{j}^{n-1}}{(\Delta t)^{2}}, \quad \ddot{y}_{j}{ }^{n}=\frac{y_{j}^{n+1}-2 y_{j}^{n}+y_{j}^{n-1}}{(\Delta t)^{2}} \\
\quad(j=1,2, \cdots s-1) .
\end{gathered}
$$

It is assumed that the boundary and initial conditions are known. These are given in eqs. (2.7) and (2.8), respectively. The system of equations summarized above, consisting of eqs. (2.2), (2.6), (2.7), (2.8), (3.1), (3.2), (3.3), (3.4) with the auxiliary equations $(2.1),(2.3),(2.4),(2.5)$, completely describe the motion of the cable.

The computational procedure, as developed in detail in the remainder of this section, consists of an algorithm to determine the values $x_{j}^{n+1}, y_{j}^{n+1}, \dot{x}_{j}^{n+1}, \dot{y}_{j}^{n+1}$ (at time $t=t^{n+1}=t^{n}+\Delta t$ and $t^{n+1}=t^{n}+\frac{1}{2} \Delta t$ ) from known values $x_{j}^{n}, y_{j}^{n}$, $\dot{x}_{j}^{n-1}, \dot{y}_{j}^{n-t}$ (at time $t=t^{n}$ and $t^{n-1}$ ). It is convenient to divide this algorithm in two phases. The first phase involves the determination of a tentative (but consistent) set of tensions for all segments, and numerical integration of the equations of motion to predict the coordinates one step ahead; the second phase involves the evaluation of small discrepancies in the constraint equations from which a set of first-order corrections to the tensions can be obtained, and integration of the equations a second time to obtain accurate values of the coordinates.

Phase 1. Using eqs. (2.2) and (3.2), ( $3 s-2$ equations), we compute the $(3 s-2)$ unknown variables $\ddot{x}_{j}{ }^{n}, \ddot{y}_{j}{ }^{n}(j=1,2, \cdots s-1)$ and $T_{j-1}^{n}(j=1,2, \cdots s)$. We now use eqs. (3.3) and (3.4), (4s - 4 equations); to compute the $(4 s-4)$ variables at the next time step, $x_{j}^{n+1}, y_{j}^{n+1}, \dot{x}_{j}^{n+1}, \dot{y}_{j}^{n+1}(j=1,2 \cdots s-1)$. These are considered to be only tentative values (denoted in subsequent text by use of the tilde).

Phase 2. To obtain improved values of the tensions $T_{j-\frac{1}{3}}^{n}(j=1,2, \cdots s)$ and the quantities $\ddot{x}_{j}^{n}, \ddot{y}_{j}{ }^{n}, x_{j}^{n+1}, y_{j}^{n+1}, \dot{x}_{j}^{n+1}, \dot{y}_{j}^{n+1}$ (a total of $(7 s-6)$ quantities) we use the system of equations (2.2), (2.6) and (3.3), (3.t), consisting of $(7 s-6)$ equations. However, since eqs. (2.6) are not linear but quadratic in the unknowns $x_{j}$ and $y_{j}$, an explicit solution is impractical to obtain. For this reason a computation algorithm based on the Newton-Raphson method of successive approximations 
is developed. A detailed discussion of the computational procedure used in this problem is given in the sections which follow.

B. Determatiox of Tentative Values of Texsioxs. The system of equations (2.2) may be regarded as a set of $(2 s-2)$ linear equations in the variables $\tilde{x}_{j}$ and $\ddot{y}_{j}$ (accelerations) and may be solved directly for these variables. If we designate

$$
\begin{aligned}
L_{j} & =(\Delta t)^{2} I_{j} /\left(I_{j} J_{j}-K_{j}^{2}\right) \\
M_{j} & =(\Delta t)^{2} J_{j} /\left(I_{j} J_{j}-K_{j}^{2}\right) \\
N_{j} & =(\Delta t)^{2} K_{j} /\left(I_{j} J_{j}-K_{j}^{2}\right),
\end{aligned}
$$

then the equations of motion (2.2) can be reduced to:

$$
\begin{aligned}
& \ddot{x}_{j}=\left[R_{j} T_{j+\xi}-P_{j} T_{j-\xi}+U_{j}\right] /(\Delta t)^{2} \\
& \ddot{y}_{j}=\left[S_{j} T_{j+\xi}-Q_{j} T_{j-1}+V_{j}\right] /(\Delta t)^{2}
\end{aligned}
$$

where

$$
\begin{aligned}
& P_{j}=M_{j} \cos \theta_{j-1}+N_{j} \sin \theta_{j-1} \\
& Q_{j}=N_{j} \cos \theta_{j-1}+L_{j} \sin \theta_{j-1} \\
& R_{j}=M_{j} \cos \theta_{j+1}+N_{j} \sin \theta_{j+1} \\
& S_{j}=N_{j} \cos \theta_{j+1}+L_{j} \sin \theta_{j+1} \\
& U_{j}=M_{j} X_{j}+N_{j} Y_{j} \\
& V_{j}=N_{j} X_{j}+L_{j} Y_{j} .
\end{aligned}
$$

We observe that eq. (3.2) involves positions, velocities, and accelerations. As is often the case with finite-difference procedures, it proves to be convenient to compute positions and accelerations at the mesh points while velocities are found at the midpoints in time. For this reason we shall use a modified form obtained by evaluating eq. (3.2) at $t=t^{n}$ and at $t=t^{n-1}$, and then adding the two results together, namely,

$$
\begin{aligned}
& \left(x_{j}^{n}-x_{j-1}^{n}\right)\left(\ddot{x}_{j}^{n}-\ddot{x}_{j-1}^{n}\right)+\left(y_{j}^{n}-y_{j-1}^{n}\right)\left(\ddot{y}_{j}^{n}-\dot{y}_{j-1}^{n}\right) \\
& \quad+\left(x_{j}^{n-1}-x_{j-1}^{n-1}\right)\left(\bar{x}_{j}^{n-1}-\bar{x}_{j-1}^{n-1}\right)+\left(y_{j}^{n-1}-y_{j-1}^{n-1}\right)\left(\dot{y}_{j}^{n-1}-\ddot{y}_{j-1}^{n-1}\right) \\
& \quad+2(\Delta t)^{-2}\left[\left(x_{j}^{n}-x_{j}^{n-1}\right)-\left(x_{j-1}^{n}-x_{j-1}^{n-1}\right)\right]^{2} \\
& \quad+2(\Delta t)^{-2}\left[\left(y_{j}^{n}-y_{j}^{n-1}\right)-\left(y_{j-1}^{n}-y_{j-1}^{n-1}\right)\right]^{2}=0,
\end{aligned}
$$

in which we have used the approximations,

$$
\begin{aligned}
\left(\dot{x}_{j}^{n}-\dot{x}_{j-1}^{n}\right)^{2}+\left(\dot{x}_{j}^{n-1}-\dot{x}_{j-1}^{n-1}\right)^{2} & \approx 2\left(\dot{x}_{j}^{n-1}-\dot{x}_{j-1}^{n-1}\right)^{-} \\
& \approx 2\left(\left(x_{j}^{n}-x_{j}^{n-1}\right) / \Delta t-\left(x_{j-1}^{n}-x_{j-1}^{n-1}\right) / \Delta t\right]^{2}, \\
\left(\dot{y}_{j}^{n}-\dot{y}_{j-1}^{n}\right)^{2}+\left(\dot{y}_{j}^{n-1}-\dot{y}_{j-1}^{n-1}\right)^{2} & \approx 2\left(\dot{y}_{j}^{n-1}-\dot{y}_{j-1}^{n-1}\right)^{2} \\
& \approx 2\left[\left(y_{j}^{n}-y_{j}^{n-1}\right) / \Delta t-\left(y_{j-1}^{n}-y_{j-1}^{n-1}\right) / \Delta t\right]^{2} .
\end{aligned}
$$

Note that eqs. (3.6) are linear in the accelerations. Likewise, eqs. (3.5) are linear in the tensions. Consequently, when these latter expressions are substituted for the 
acceleration components in the constraint equations (3.6), we obtain a set of conditions which are linear in the tensions, namely,

$$
\begin{aligned}
& E_{j-1}^{n} T_{j-1}^{n}-F_{j-1}^{n} T_{j-1}^{n}+G_{j-1}^{n} T_{j+1}^{n} \\
& +E_{j-1}^{n-1} T_{j-1}^{n-1}-F_{j-1}^{n-1} T_{j-\frac{1}{2}}^{n-1}+G_{j-1}^{n-1} T_{j+\frac{1}{j}}^{n-1}+H_{j-1}^{n}+H_{j-1}^{n-1} \\
& +2\left[\left(x_{j}^{n}-x_{j}^{n-1}\right)-\left(x_{j-1}^{n}-x_{j-1}^{n-1}\right)\right]^{2} \\
& \quad+2\left[\left(y_{j}^{n}-y_{j}^{n-1}\right)-\left(y_{j-1}^{n}-y_{j-1}^{n-1}\right)\right]^{2}=0
\end{aligned}
$$

where

$$
\begin{aligned}
& E_{j-1}^{n}=\left(x_{j}^{n}-x_{j-1}^{n}\right) P_{j-1}^{n}+\left(y_{j}^{n}-y_{j-1}^{n}\right) Q_{j-1}^{n} \\
& F_{j-1}^{n}=\left(x_{j}^{n}-x_{j-1}^{n}\right)\left(P_{j}^{n}+R_{j-1}^{n}\right)+\left(y_{j}^{n}-y_{j-1}^{n}\right)\left(Q_{j}^{n}+S_{j-1}^{n}\right) \\
& G_{j-1}^{n}=\left(x_{j}^{n}-x_{j-1}^{n}\right) R_{j}^{n}+\left(y_{j}^{n}-y_{j-1}^{n}\right) S_{j}^{n} \\
& H_{j-1}^{n}=\left(x_{j}^{n}-x_{j-1}^{n}\right)\left(U_{j}^{n}-U_{j-1}^{n}\right)+\left(y_{j}^{n}-y_{j-1}^{n}\right)\left(V_{j}^{n}-V_{j-1}^{n}\right) .
\end{aligned}
$$

Now assume that the solution is correct up to $t=t^{n}$. Then all quantities in (3.7) can be evaluated at once except for $T_{j-k}^{n}, T_{j-1}^{n}$ and $T_{j+1}^{n}$. The tentative values of the tensions-signified by the tildes-are determined by the following system of equations:

$$
\left[\begin{array}{rrrrrr}
-F_{0.5}^{n} & G_{0.5}^{n} & & & \\
E_{1.5}^{n} & -F_{1.5}^{n} & G_{1.5}^{n} & & \\
& E_{2.5}^{n} & -F_{2.5}^{n} & G_{2.5}^{n} . & \\
& & \cdot & \cdot & \\
& & E_{s-1.5}^{n} & -\dot{F}_{s-1.5}^{n} & G_{s-1.5}^{n} \\
& & & & E_{s-0.5}^{n} & -F_{s-0.5}^{n}
\end{array}\right]\left[\begin{array}{l}
T_{0.5}^{n} \\
T_{1.5}^{n} \\
T_{2.5}^{n} \\
\vdots \\
\dot{T}_{s-1.5}^{n} \\
T_{s-0.5}^{n}
\end{array}\right]=\left[\begin{array}{c}
-\Psi_{0.5}^{n} \\
-\Psi_{1.5}^{n} \\
-\Psi_{2.5}^{n} \\
\vdots \\
-\Psi_{s-1.5}^{n} \\
-\Psi_{s-0.5}^{n}
\end{array}\right]
$$

where

$$
\begin{aligned}
\Psi_{j-1}^{n}= & E_{j-1}^{n-1} T_{j-1}^{n-1}-F_{j-1}^{n-1} T_{j-1}^{n-1}+G_{j-1}^{n-1} T_{j+\frac{1}{j}}^{n-1}+H_{j-\frac{1}{3}}^{n-1}+H_{j-\frac{1}{j}}^{n} \\
& +2\left[\left(x_{j}^{n}-x_{j}^{n-1}\right)-\left(x_{j-1}^{n}-x_{j-1}^{n-1}\right)\right]^{2}+2\left[\left(y_{j}^{n}-y_{j}^{n-1}\right)-\left(y_{j-1}^{n}-y_{j-1}^{n-1}\right)\right]^{2} .
\end{aligned}
$$

In general, we can write (for $j=1,2, \cdots s$ )

$$
E_{j-1}^{n} T_{j-1}^{n}-F_{j-1}^{n} T_{j-1}^{n}+G_{j-1}^{n} T_{j+1}^{n}+\Psi_{j-1}^{n}=0
$$

with the conditions: $E_{0.5}^{n}=G_{s-1}^{n}=0$ for all $n$. Also, $P_{0}{ }^{n}, Q_{0}{ }^{n}, R_{0}{ }^{n}, S_{0}{ }^{n}$, and $P_{s}{ }^{n}$, $Q_{s}{ }^{n}, R_{s}{ }^{n}, S_{s}{ }^{n}=0$ for all $n$; and

$$
\begin{array}{llll}
U_{0}^{n}=(\Delta t)^{2} \ddot{x}_{0}^{n} & \text { and } & U_{s}^{n}=(\Delta t)^{2} \ddot{x}_{s}^{n} & \text { for all } n, \\
V_{0}^{n}=(\Delta t)^{2} \ddot{y}_{0}{ }^{n} & \text { and } & V_{s}^{n}=(\Delta t)^{2} \ddot{y}_{s}^{n} & \text { for all } n .
\end{array}
$$

The matrix of coefficients of the system of equations is a triple diagonal one, and it can be easily reduced to a single linear equation by elimination. Thus, we solve eq. (3.9) for $T_{j+1}^{n}$.

$$
T_{j+1}^{n}=\frac{F_{j-1}^{n}}{G_{j-1}^{n}} T_{j-1}^{n}-\frac{E_{j-1}^{n}}{G_{j-1}^{n}} T_{j-1}^{n}-\frac{\Psi_{j-1}^{n}}{G_{j-1}^{n}}
$$


Now we express each tension as a linear function of $T_{0.5}^{n}$ (the tension in the first segment) as follows:

$$
T_{j+j}^{n}=\alpha_{j+1}^{n} T_{0.5}^{n}+\beta_{j+j}^{n}
$$

and we arrive at the following recursion formulas for $\alpha_{j+j}^{n}$ and $\beta_{j+1}^{n}$, namely,

$$
\begin{aligned}
& \alpha_{j+1}^{n}=\left(F_{j-1}^{n} \alpha_{j-1}^{n}-E_{j-1}^{n} \alpha_{j-1}^{n}\right) / G_{j-1}^{n} \\
& \beta_{j+1}^{n}=\left(F_{j-1}^{n} \beta_{j-1}^{n}-E_{j-1}^{n} \beta_{j-1}^{n}-\Psi_{j-1}^{n}\right) / G_{j-1}^{n}
\end{aligned}
$$

with the conditions

$$
\begin{array}{lll}
\alpha_{0.5}^{n}=1, & \alpha_{-0.5}^{n}=0 & \text { for all } n, \\
\beta_{0.5}^{n}=0, & \beta_{-0.5}^{n}=0 & \text { for all } n .
\end{array}
$$

Starting with $j=1$, we evaluate $\alpha_{j+3}^{n}$ and $\beta_{j+1}^{n}$ recursively up to $j=s-1$. We then find $T_{0.5}^{n}$ from the last equation of the system.

$$
T_{0.5}^{n}=-\frac{\left(F_{s-1}^{n} \beta_{s-1}^{n}-E_{s-1}^{n} \beta_{s-1}^{n}-\Psi_{s-1}^{n}\right)}{\left(F_{s-\frac{1}{3}}^{n} \alpha_{s-\frac{1}{1}}^{n}-E_{s-\frac{1}{3}}^{n} \alpha_{s-1}^{n}\right)} .
$$

C. Computation of Tentative Coordinates. In order to solve eqs. (3.5) numerically, we replace $\ddot{x}_{j}{ }^{n}$ and $\ddot{y}_{j}{ }^{n}$ by their simplest central-difference approximations, eq. (3.4), namely,

$$
\begin{aligned}
& \ddot{x}_{j}{ }^{n}=\left(x_{j}^{n+1}-2 x_{j}{ }^{n}+x_{j}^{n-1}\right) /(\Delta t)^{2} \\
& \ddot{y}_{j}{ }^{n}=\left(y_{j}^{n+1}-2 y_{j}{ }^{n}+y_{j}^{n-1}\right) /(\Delta t)^{2} .
\end{aligned}
$$

Now we solve for $x_{j}^{n+1}$ and $y_{j}^{n+1}$, considering these as tentative values subject to a slight modification in order to satisfy a system of constraints. Thus we write

$$
\begin{aligned}
& \bar{x}_{j}^{n+1}=2 x_{j}^{n}-x_{j}^{n-1}-P_{j}^{n} T_{j-1}^{n}+R_{j}^{n} T_{j+1}^{n}+U_{j}^{n} \\
& \tilde{y}_{j}^{n+1}=2 y_{j}^{n}-y_{j}^{n-1}-Q_{j}{ }^{n} T_{j-1}^{n}+S_{j}^{n} T_{j+1}^{n}+V_{j}^{n} .
\end{aligned}
$$

The quantities $P_{j}{ }^{n}, Q_{j}{ }^{n}, R_{j}{ }^{n}, S_{j}{ }^{n}, U_{j}{ }^{n}$ and $V_{j}{ }^{n}$ are the same as were used to set up the coefficient matrix for the tensions, and the values for $T_{j-\xi}^{n}$ and $T_{i+1}^{n}$ are obtained from (3.11).

D. Determination of Improved Values of Tensions. Next, we determine the set of corrections $\delta T_{j-1}^{n}$ to be applied to the tensions $T_{j-1}^{n}$ in order that the values of $x_{i}^{n+1}$ and $y_{j}^{n+1}$ should also satisfy the inextensibility condition (2.6). For this purpose we define the function

$$
\Omega_{j-1}^{n+1} \equiv \frac{1}{2}\left[\left(x_{i}^{n+1}-x_{j-1}^{n+1}\right)^{2}+\left(y_{j}^{n+1}-y_{j-1}^{n+1}\right)^{2}-l_{j-1}^{2}\right]
$$

which measures the discrepancy in the distance between the extrapolated positions of pairs of adjacent stations. We observe from eqs. (3.15) - with the tildes suppressed-that $x_{j}^{n+1}$ and $y_{j}^{n+1}$ are functions of the tensions. Consequently, $\Omega_{j-i}^{n+1}$ may also be expressed as a function of the tensions. This enables us to write the system of constraints to which the tensions are subject as follows:

$$
\Omega_{j-1}^{n+1}=\Omega_{j-1}^{n+1}\left\{T_{j-1}^{n}, T_{j-1}^{n}, T_{j+1}^{n}\right\}=0 \quad(j=1,2, \cdots s)
$$

since $\Omega_{j-j}^{n+1}$ vanishes when the inextensibility condition is obeyed. 
Now let

$$
T_{j+1}^{n}=T_{j+1}^{n}+\delta T_{j+1}^{n},
$$

and expand $\Omega_{j-1}^{n+1}$ in a Taylor series about the point $\left\{T_{j-1}^{n}, T_{j-j}^{n}, T_{j+1}^{n}\right\}$. Thus, we obtain

$$
\begin{aligned}
\Omega_{j-j}^{n+1}=\tilde{\Omega}_{j-1}^{n+1}+\frac{\partial \tilde{\Omega}_{j-1}^{n+1}}{\partial T_{j-1}^{n}} \delta T_{j-1}^{n}+\frac{\partial \tilde{\Omega}_{j-3}^{n+1}}{\partial T_{j-3}^{n}} \delta T_{j-1}^{n} \\
+\frac{\partial \tilde{\Omega}_{j-j}^{n+1}}{\partial T_{j+3}^{n+3}} \delta T_{j+1}^{n}+\text { higher order terms, }
\end{aligned}
$$

where

$$
\begin{aligned}
\tilde{\Omega}_{j-1}^{n+1} & \equiv \Omega_{j-\frac{1}{3}}^{n+1}\left\{T_{j-1}^{n}, T_{j-1}^{n}, T_{j+1}^{n}\right\} \\
& =\frac{1}{2}\left[\left(\bar{x}_{j}^{n+1}-\tilde{x}_{j-1}^{n+1}\right)^{2}+\left(\tilde{y}_{j}^{n+1}-\tilde{y}_{j-1}^{n+1}\right)^{2}-l_{j-1}^{2}\right] .
\end{aligned}
$$

Provided that the tentative values $T_{j-\frac{1}{3}}^{n}$ are sufficiently close to the correct values $T_{j-\frac{1}{3}}^{n}$, we may neglect the higher order terms in the expansion (3.19), and thereby obtain a system of $s$ linear equations for the differential corrections $\delta T_{j-1}^{n}$. These equations have the same form as the previous system (3.8) for determining $T_{j-1}^{n}$, namely,

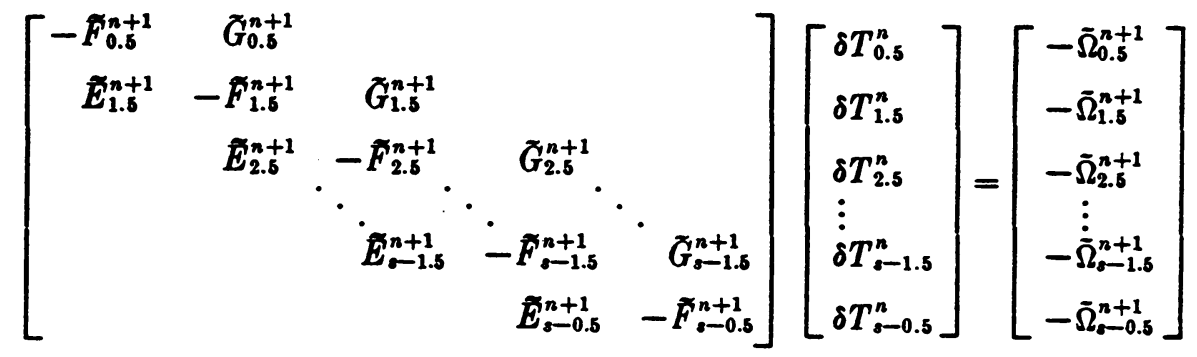

the general expression being (for $j=1,2, \cdots s$ )

$$
\tilde{E}_{j-\frac{1}{j}}^{n+1} \delta T_{j-1}^{n}-\tilde{F}_{j-\frac{1}{j}}^{n+1} \delta T_{j-\frac{1}{2}}^{n}+\bar{G}_{j-\frac{1}{j}}^{n+1} \delta T_{j+\frac{1}{j}}^{n}+\tilde{\Omega}_{j-\frac{1}{j}}^{n+1}=0
$$

where

$$
\begin{aligned}
& \tilde{E}_{j-\frac{1}{3}}^{n+1}=\frac{\partial \tilde{\Omega}_{j-1}^{n+1}}{\partial T_{j-1}^{n}}=\left(\tilde{x}_{j}^{n+1}-\tilde{x}_{j-1}^{n+1}\right) P_{j-1}^{n}+\left(\tilde{y}_{j}^{n+1}-\tilde{y}_{j-1}^{n+1}\right) Q_{j-1}^{n} \\
& \tilde{F}_{j-\frac{1}{3}}^{n+1}=-\frac{\partial \tilde{\Omega}_{j-1}^{n+1}}{\partial T_{j-1}^{n}}=\left(\tilde{x}_{j}^{n+1}-\tilde{x}_{j-1}^{n+1}\right)\left(P_{j}^{n}+R_{j-1}^{n}\right)+\left(\tilde{y}_{i}^{n+1}-\tilde{y}_{j-1}^{n+1}\right)\left(Q_{j}^{n}+S_{j-1}^{n}\right) \\
& \tilde{G}_{j-\frac{1}{3}}^{n+1}=\frac{\partial \tilde{\Omega}_{j-1}^{n+1}}{\partial T_{j+\frac{1}{3}}^{n}}=\left(\tilde{x}_{j}^{n+1}-\tilde{x}_{j-1}^{n+1}\right) R_{j}^{n}+\left(\tilde{y}_{j}^{n+1}-\tilde{y}_{j-1}^{n+1}\right) S_{j}^{n}
\end{aligned}
$$

with the conditions: $\widehat{E}_{0.5}^{n+1}=G_{j-1}^{n+1}=0$ for all $n$, and the quantities $P_{j}^{n}, Q_{j}{ }^{n}, R_{j}^{n}$ and $S_{j}{ }^{n}$ being the same as in (3.7).

The system (3.20) can be solved in a manner completely analogous to the. solution of the system (3.8). Thus, we write

$$
\delta T_{j+\xi}^{n}=\alpha_{j+\xi}^{n} \delta T_{0.8}^{n}+\lambda_{j+\xi}^{n}
$$


and obtain the following recursion formulas:

$$
\begin{aligned}
\kappa_{j+1}^{n} & =\left(\widetilde{F}_{j-1}^{n+1} \kappa_{j-1}^{n}-\widetilde{E}_{j-1}^{n+1} \kappa_{j-1}^{n}\right) / G_{j-1}^{n+1} \\
\lambda_{j+1}^{n} & =\left(\widetilde{F}_{j-1}^{n+1} \lambda_{j-1}^{n}-\tilde{E}_{j-j}^{n+1} \lambda_{j-1}^{n}-\tilde{\Omega}_{j-1}^{n+1}\right) / G_{j-1}^{n+1}
\end{aligned}
$$

with the conditions

$$
\begin{array}{lll}
\kappa_{0.5}^{n}=1, & \kappa_{-0.5}^{n}=0 & \text { for all } n, \\
\lambda_{0.5}^{n}=0, & \lambda_{0.5}^{n}=0 & \text { for all } n .
\end{array}
$$

Finally, the last equation of the system enables us to solve for $\delta T_{0.5}^{n}$. The result is

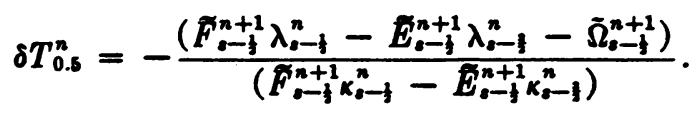

We can now obtain the corrected values of the tension in each segment. Thus,

$$
\begin{aligned}
T_{j-1}^{n} & =T_{j-1}^{n}+\delta T_{j-1}^{n} \\
& =T_{j-1}^{n}+\kappa_{j-j}^{n} \delta T_{0.5}^{n}+\lambda_{j-1}^{n} .
\end{aligned}
$$

E. Computation of Improved Coordinates. The corrected values of the coordinates are found using ec(s. (3.15) - but this time with the tildes suppressednamely:

$$
\begin{aligned}
& x_{j}^{n+1}=2 x_{j}^{n}-x_{j}^{n-1}-P_{j}^{n} T_{j-1}^{n}+R_{j}^{n} T_{j+1}^{n}+U_{j}^{n} \\
& y_{i}^{n+1}=2 y_{j}^{n}-y_{j}^{n-1}-Q_{j}^{n} T_{j-1}^{n}+S_{j}^{n} T_{i+1}^{n}+V_{j}^{n} .
\end{aligned}
$$

For solution on an automatic computer, it is more convenient to express eqs. (3.26) in terms of corrections to be added to the tentative values of the coordinates. That is,

$$
\begin{aligned}
\delta x_{j}^{n+1} & =-P_{j}^{n} \delta T_{j-1}^{n}+R_{j}^{n} \delta T_{j+1}^{n} \\
\delta y_{i}^{n+1} & =-Q_{j}^{n} \delta T_{j-1}^{n}+S_{j}^{n} \delta T_{j+1}^{n} .
\end{aligned}
$$

Then the corrected coordinates are given by:

$$
\begin{aligned}
& x_{j}^{n+1}=\tilde{x}_{j}^{n+1}+\delta x_{i}^{n+1} \\
& y_{i}^{n+1}=\tilde{y}_{j}^{n+1}+\delta y_{j}^{n+1} .
\end{aligned}
$$

F. Special Form of Equations for Computing First Time Step. We assume that the initial velocity components are zero at each station, and we obtain the initial coordinates from the equations for static equilibrium of the line. Since $\dot{x}_{j}{ }^{0}$ and $\dot{y}_{j}{ }^{0}=0$, eq. (3.2) reduces to

$$
\left(x_{j}^{0}-x_{j-1}^{0}\right)\left(\ddot{x}_{j}^{0}-\ddot{x}_{i-1}^{0}\right)+\left(y_{j}^{0}-y_{j-1}^{0}\right)\left(\ddot{y}_{j}^{0}-\ddot{y}_{j-1}^{0}\right)=0
$$

and, on substituting the expressions (3.5), we find that the tensions are subject to the constraint

$$
E_{j-1}^{0} T_{j-1}^{0}-F_{j-1}^{0} T_{j-1}^{0}+G_{j-1}^{0} T_{j+1}^{0}+H_{j-1}^{0}=0 .
$$

Comparing this with eq. (3.9), we see that

$$
\Psi_{i-\}}^{0}=H_{i-\}}^{0} \quad(j=1,2, \cdots s) .
$$


The system of equations (3.8) is then solved in the usual way to get the proper initial tensions $T_{j-1}^{0}$.

To obtain tentative values for the coordinates at $t=t^{2}$, we make use of their Taylor series expansions about the point $t=t^{0}$, namely:

$$
\begin{aligned}
& x_{j}{ }^{1}=x_{j}{ }^{0}+(\Delta t) \dot{x}_{j}{ }^{0}+\frac{1}{2}(\Delta t)^{2} \ddot{x}_{j}{ }^{0}+\cdots \\
& y_{j}{ }^{1}=y_{j}{ }^{0}+(\Delta t) \dot{y}_{j}{ }^{0}+\frac{1}{2}(\Delta t)^{2} \ddot{y}_{j}{ }^{0}+\cdots
\end{aligned}
$$

Taking $\dot{x}_{j}{ }^{0}$ and $\dot{y}_{j}{ }^{0}=0$, and substituting eqs. (3.5) for $\ddot{x}_{j}{ }^{0}$ and $\ddot{y}_{j}{ }^{0}$, we find

$$
\begin{aligned}
& \tilde{x}_{j}{ }^{1}=x_{j}{ }^{0}+\frac{1}{2}\left[-P_{j}{ }^{0} \tilde{T}_{j-\frac{1}{2}}^{0}+R_{j}{ }^{0} \tilde{T}_{j+1}^{0}+U_{j}^{0}\right] \\
& \tilde{y}_{j}{ }^{1}=y_{j}{ }^{0}+\frac{1}{2}\left[-Q_{j}{ }^{0} \tilde{T}_{j-1}^{0}+S_{j}{ }^{0} \tilde{T}_{j+1}^{0}+V_{j}^{0}\right] .
\end{aligned}
$$

The corrections to the tensions are then determined by the system of equations (3.20) in the usual manner. Finally, the corrections to the coordinates are computed as follows:

$$
\begin{aligned}
\delta x_{j}{ }^{1} & =\frac{1}{2}\left[-P_{j}{ }^{0} \delta T_{j-\frac{1}{2}}^{0}+R_{j}{ }^{0} \delta T_{j+\frac{1}{3}}^{0}\right] \\
\delta y_{j}{ }^{1} & =\frac{1}{2}\left[-Q_{j}{ }^{0} \delta T_{j-\frac{1}{2}}^{0}+S_{j}{ }^{0} \delta T_{j+\frac{1}{3}}^{0}\right]
\end{aligned}
$$

and the corrected coordinates are given by:

$$
\begin{aligned}
& x_{j}{ }^{1}=\tilde{x}_{j}{ }^{1}+\delta x_{j}{ }^{1} \\
& y_{j}{ }^{1}=\tilde{y}_{j}{ }^{1}+\delta y_{j}{ }^{1} .
\end{aligned}
$$

4. Analysis of Numerical Stability. In order to obtain a valid solution of the system of partial differential equations governing the generalized motion of a cable, it is necessary to insure the stability (in the sense discussed in [2], [3], [4]) of the equivalent finite-difference system (2.2), (2.6), (3.3), (3.4). In this section we will derive the criteria for stability of this system of equations. We will also show that whereas the system of finite-difference equations (2.2), (2.6), (3.3), (3.4) is stable for sufficiently small time intervals $\Delta t$, the system (2.2), (3.2), (3.3), (3.4) is always unstable. This characteristic of the latter system has led to the abandonment of this simpler set of equations in favor of the more difficult nonlinear system (2.2), (2.6), (3.3), (3.4).

In order to determine the stability of a system of finite-difference equations, we study the growth of a small disturbance or perturbation. The conditions for stability are said to be satisfied if the amplitude of a small disturbance, introduced at any time, $t$, in any of the dependent variables, does not increase exponentially with successive time steps. This condition may be stated as follows:

If $\delta F(s, t)$ and $\delta F(s, t+\Delta t)$ are values of a variation (or perturbation) in any of the dependent variables $x, y, T$ in the system, then it is said to be stable provided $|\delta F(s, t+\Delta t) / \delta F(s, t)| \leqq 1$. We introduce perturbations $\delta x, \delta y, \delta T$ in the dependent variables $x, y, T$, respectively. To simplify the stability investigation, we shall omit the terms pertaining to the suspended weights as well as all terms involving virtual inertia. From eqs. (2.2), (2.6), (3.3), and (3.4) we obtain the following variational system of equations:

$$
\begin{aligned}
m_{j} \delta \ddot{x}_{j}=T_{j+\frac{1}{2}} \delta \cos \theta_{j+\frac{1}{3}} & -T_{j-\frac{1}{1}} \delta \cos \theta_{j-\frac{1}{1}}+\cos \theta_{j+1} \delta T_{j+1} \\
& -\cos \theta_{j-\frac{1}{1}} \delta T_{j-\frac{1}{2}}-\frac{1}{2}\left[D_{j+\frac{1}{2}} \delta \sin \theta_{j+\frac{1}{1}}+D_{j-\frac{1}{1}} \delta \sin \theta_{j-\frac{1}{1}}\right. \\
& \left.+\sin \theta_{j+\frac{1}{2}} \delta D_{j+1}+\sin \theta_{j-\frac{1}{2}} \delta D_{j-\frac{1}{1}}\right]
\end{aligned}
$$




$$
\begin{aligned}
& m_{j} \delta \tilde{y}_{j}=T_{j+\xi} \delta \sin \theta_{j+\xi}-T_{j-1} \delta \sin \theta_{j-\xi}+\sin \theta_{j+\xi} \delta T_{j+\xi} \\
& -\sin \theta_{j-1} \delta T_{j-3}+\frac{1}{2}\left[D_{j+\}} \delta \cos \theta_{j+1}+D_{j-1} \delta \cos \theta_{j-1}\right. \\
& \left.+\cos \theta_{j+1} \delta D_{j+1}+\cos \theta_{j-1} \delta D_{j-1}\right] \text {, } \\
& \cos \theta_{j+\frac{1}{2}} \delta \cos \theta_{j+\frac{1}{2}}+\sin \theta_{j+\frac{1}{3}} \delta \sin \theta_{j+\frac{1}{3}}=0 \text {, }
\end{aligned}
$$

where

$$
\begin{aligned}
& \delta D_{j+1}=-2 f_{j+1}^{D}\left|q_{j+1}\right| \delta q_{j+1}, \\
& \delta q_{j+1}=-\frac{1}{2}\left[\left(\dot{x}_{j+1}-c\right)+\left(\dot{x}_{j}-c\right)\right] \delta \sin \theta_{j+1}+\frac{1}{2}\left(\dot{y}_{j+1}+\dot{y}_{j}\right) \delta \cos \theta_{j+1} \\
& \text { and where } \\
& -\frac{1}{2} \sin \theta_{j+3}\left(\delta \dot{x}_{j+1}+\delta \dot{x}_{j}\right)+\frac{1}{2} \cos \theta_{j+1}\left(\delta \dot{y}_{j+1}+\delta \dot{y}_{j}\right) ;
\end{aligned}
$$

$$
\begin{aligned}
\delta \cos \theta_{j+\frac{1}{3}}=\left(\delta x_{j+1}-\delta x_{j}\right) / l_{j+1}, & \delta \sin \theta_{j+1}=\left(\delta y_{j+1}-\delta y_{j}\right) / l_{j+1} ; \\
\delta \dot{x}_{j}^{n-1}=\left(\delta x_{j}^{n}-\delta x_{i}^{n-1}\right) / \Delta t, & \delta \dot{y}_{j}^{n-1}=\left(\delta y_{j}^{n}-\delta y_{j}^{n-1}\right) / \Delta t ; \\
\delta \ddot{x}_{j}^{n}=\left(\delta x_{j}^{n+1}-2 \delta x_{j}^{n}+\delta x_{i}^{n-1}\right) /(\Delta t)^{2}, & \delta \dot{y}_{j}^{n}=\left(\delta y_{j}^{n+1}-2 \delta y_{j}^{n}+\delta y_{j}^{n-1}\right) /(\Delta t)^{2} .
\end{aligned}
$$

We will assume in this analysis that within a small region in the $(s, t)$ plane the coefficients $\left(T_{j}{ }^{n}, \cos \theta_{j}{ }^{n}, D_{j}{ }^{n}\right.$, etc. $)$ of the variational functions vary only slightly and hence may be treated as constants. We will denote these simply by $T, \cos \theta$, $D$, etc., omitting the indices. A solution of the system of equations (4.1) can then be obtained in the form

$$
\begin{aligned}
& \delta x_{j}{ }^{n}=a e^{i \beta j+\alpha n \Delta t} \\
& \delta y_{j}{ }^{n}=b e^{i \beta j+\alpha \Delta \Delta t} \\
& \delta T_{j}{ }^{n}=c e^{i \beta j+\alpha n \Delta t}
\end{aligned}
$$

where $a, b, c$ are real constants and $\alpha$ complex. Substituting in eq. (4.1), we obtain a system of linear homogeneous equations for the quantities $a, b$ and $c$ which has a non-trival solution, provided the determinant of the coefficients is identically zero. After some algebraic simplifications, the determinant of the coefficients may be written in the form

where

$$
\left|\begin{array}{ccc}
F-A \sin \theta & D^{\prime}+B \sin \theta & \cos \theta \\
-D^{\prime}+A \cos \theta & F-B \cos \theta & \sin \theta \\
\cos \theta & \sin \theta & 0
\end{array}\right|=0
$$

$$
\begin{aligned}
A & =f|q|\left[2 i \dot{y} \sin \beta-(l \sin \theta / \Delta t)(1+\cos \beta)\left(1-\lambda^{-1}\right)\right] \\
B & =f|q|\left[2 i(\dot{x}-c) \sin \beta-(l \cos \theta / \Delta t)(1+\cos \beta)\left(1-\lambda^{-1}\right)\right] \\
D^{\prime} & =i D \sin \beta \\
F & =m l \xi /(\Delta t)^{2}+4 T \sin ^{2}(\beta / 2)
\end{aligned}
$$

and where

$$
\lambda=e^{\alpha \Delta t}, \quad \xi=\left(\lambda-2+\lambda^{-1}\right) .
$$

Multiplying the elements of the determinant and simplifying, we obtain

$$
A \sin \theta+B \cos \theta-F=0
$$


But,

$$
A \sin \theta+B \cos \theta=f|q|\left[(2 i \sin \beta) p-(l / \Delta t)(1+\cos \beta)\left(1-\lambda^{-1}\right)\right]
$$

where

$$
p=(\dot{x}-c) \cos \theta+\dot{y} \sin \theta
$$

i.e., the tangential component of the velocity of the cable (relative to the medium). Substituting in equation (4.3), we finally obtain the characteristic equation of the variational system (4.1), namely,

$$
\begin{aligned}
m l \lambda^{2}+ & \{f|q| \Delta t[l(1+\cos \beta)-p \Delta t(2 i \sin \beta)] \\
& \left.+4 T(\Delta t)^{2} \sin ^{2}(\beta / 2)-2 m l\right\} \lambda+[m l-f|q| l \Delta t(1+\cos \beta)]=0 .
\end{aligned}
$$

Now, comparing the first and second terms of the coefficient of $\lambda$, we find that the second term is negligibly small provided $2 p \Delta t \ll l$, i.e., the tangential distance traversed by the cable in one time step is very small compared with the length of the cable segment. Since this is usually the case and, at any rate, can always be satisfied by taking the time step sufficiently small, we will omit this term from our subsequent analysis.

For the case of negligible drag, i.e., $f=0$, approximately, we obtain from eq. (4.4)

$$
\lambda^{2}+\left[4 T \sin ^{2}(\beta / 2)(\Delta t)^{2} / m l-2\right] \lambda+1=0 .
$$

In order for the solution to be stable, the conditions $\left|\lambda_{1}\right| \leqq 1$ and $\left|\lambda_{2}\right| \leqq 1$ must both be satisfied. But if $\lambda_{1}$ is a solution of (4.5), then $\lambda_{2}=1 / \lambda_{1}$ is also a solution. It follows that the conditions for stability can be satisfied only if $\left|\lambda_{2}\right|=\left|1 / \lambda_{1}\right|=$ $\left|\lambda_{1}\right|=1$. Now, let $\lambda_{1}=\cos \gamma+i \sin \gamma, \lambda_{2}=\cos \gamma-i \sin \gamma=1 / \lambda_{1}$; then, $\left|\lambda_{1}+\lambda_{2}\right|=|2 \cos \gamma| \leqq 2$. Furthermore, from eq. (4.5) we have

$$
\lambda_{1}+\lambda_{2}=2-\frac{4 T \sin ^{2}(\beta / 2)(\Delta t)^{2}}{m l} .
$$

We thus obtain the inequality

or

$$
\left|2-4 T \sin ^{2}(\beta / 2)(\Delta t)^{2} / m l\right| \leqq 2,
$$

$$
T \sin ^{2}(\beta / 2)(\Delta t)^{2} / m l \leqq 1 .
$$

This requirement is tantamount to the condition

$$
\Delta t \leqq \sqrt{\frac{m l}{T}}=\frac{l}{\text { velocity of transverse wave }} .
$$

In the more general case, allowing for finite drag, eq. (4.4) may be reduced to (after neglecting the second term of the coefficient of $\lambda$ )

$$
\begin{aligned}
m \lambda^{2}+\left[2 f|q| l \Delta t \cos ^{2}(\beta / 2)+4 T(\Delta t)^{2}\right. & \left.\sin ^{2}(\beta / 2)-2 m l\right] \lambda \\
& +\left[m l-2 f|q| l \Delta t \cos ^{2}(\beta / 2)\right]=0 .
\end{aligned}
$$

This equation is more difficult to analyze. However, it is possible to show that both $\left|\lambda_{1}\right| \leqq 1$ and $\left|\lambda_{2}\right| \leqq 1$, provided that we have chosen 


$$
\begin{aligned}
& \Delta t \leqq \sqrt{\frac{m l}{T}}, \quad \text { and } \\
& \Delta t \leqq \frac{m}{f|q|}
\end{aligned}
$$

These conditions (4.8) are both necessary and sufficient to insure stability. $\dagger$

We will now show that the replacement of eq. (2.6) by its differentiated form (3.2) results in an unstable system; and that, furthermore, the use of any time interval $\Delta t$, no matter how small, does not change the unstable character of the equations. It will suffice to show that this condition exists in the case when the drag is negligible, i.e., $f=0$. The variational equation corresponding to eq. (3.2) is,

$$
\begin{aligned}
& \left(x,-x_{j-1}\right)\left(\delta \ddot{x}_{j}-\delta \ddot{x}_{j-1}\right)+\left(\ddot{x}_{j}-\ddot{x}_{j-1}\right)\left(\delta x_{j}-\delta x_{j-1}\right) \\
& +2\left(\dot{x}_{j}-\dot{x}_{j-1}\right)\left(\delta \dot{x}_{j}-\delta \dot{x}_{j-1}\right)+\left(y_{j}-y_{j-1}\right)\left(\delta \ddot{y}_{j}-\delta \ddot{y}_{j-1}\right) \\
& \quad+\left(\ddot{y}_{j}-\ddot{y}_{j-1}\right)\left(\delta y_{j}-\delta y_{j-1}\right)+2\left(\dot{y}_{j}-\dot{y}_{j-1}\right)\left(\delta \dot{y}_{j}-\delta \dot{y}_{j-1}\right)=0 .
\end{aligned}
$$

Substituting appropriate values for $\delta x$ and $\delta y$ and neglecting terms containing $f$, we find that the determinant equation (4.2) is replaced by

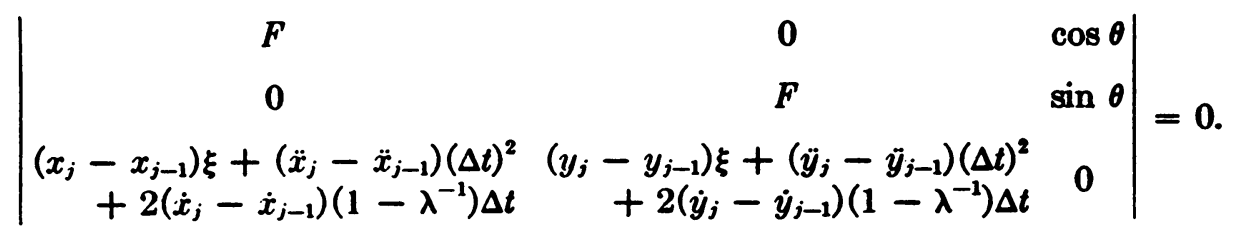

Multiplying the elements of the determinant, we obtain

$$
\begin{aligned}
& F \cos \theta\left[\left(x_{j}-x_{j-1}\right) \xi+\left(\ddot{x}_{j}-\ddot{x}_{j-1}\right)(\Delta t)^{2}+2\left(\dot{x}_{j}-\dot{x}_{j-1}\right)\left(1-\lambda^{-1}\right) \Delta t\right] \\
& +F \sin \theta\left[\left(y_{j}-y_{j-1}\right) \xi+\left(\ddot{y}_{j}-\ddot{y}_{j-1}\right)(\Delta t)^{2}+2\left(\dot{y}_{j}-\dot{y}_{j-1}\right)\left(1-\lambda^{-1}\right) \Delta t\right]=0 .
\end{aligned}
$$

Equating

$$
\cos \theta=\left(x_{j}-x_{j-1}\right) / l, \quad \sin \theta=\left(y_{j}-y_{j-1}\right) / l ;
$$

and using the relations (3.1) and (3.2), we obtain in place of eq. (4.10)

$$
F\left\{l^{2} \xi-\left[\left(\dot{x}_{j}-\dot{x}_{j-1}\right)^{2}+\left(\dot{y}_{j}-\dot{y}_{j-1}\right)^{2}\right](\Delta t)^{2}\right\}=0 \text {. }
$$

In this case the characteristic equation is a quartic, and it is necessary that the absolute values of all four roots be $\leqq 1$ to insure the numerical stability of the system. Thus, we must examine the roots of both factors of eq. (4.11), namely,

$$
F=0
$$

and

$$
l^{2} \xi-\left[\left(\dot{x}_{j}-\dot{x}_{j-1}\right)^{2}+\left(\dot{y}_{j}-\dot{y}_{j-1}\right)^{2}\right](\Delta t)^{2}=0 .
$$

It can be shown that eq. (4.12) is equivalent to the criterion (4.6) which can

† When the terms for the virtual inertia and suspended weights are included in the stability analysis, it is found that the quantity $m$ in eq. (4.8) should be replaced by the expression $m+m^{*}+e+\rho\left(V^{x} \sin ^{2} \theta+V^{Y} \cos ^{2} \theta\right)$. 
be satisfied provided

$$
\Delta t \leqq \sqrt{\frac{m l}{T}}
$$

However, the pair of roots associated with the second factor of (4.11) cannot satisfy the stability condition for any finite $\Delta t$ because eq. (4.13) requires that

$$
\xi=\left[\left(\dot{x}_{j}-\dot{x}_{j-1}\right)^{2}+\left(\dot{y}_{j}-\dot{y}_{j-1}\right)^{2}\right](\Delta t)^{2} / l^{2},
$$

an inherently positive quantity. This conclusion follows as a result of the definition $\xi=\lambda-2+\lambda^{-1}$. If $\lambda_{3}$ is a root of equation (4.13), then $\lambda_{4}=1 / \lambda_{3}$ is also a root of this equation. As before it follows that for stability $\left|\lambda_{3}\right| \leqq 1$ and $\left|\lambda_{4}\right|=\left|1 / \lambda_{3}\right| \leqq 1$. Hence, $\left|\lambda_{3}\right|=\left|\lambda_{4}\right|=1$. Let $\lambda_{3}=\cos \gamma+i \sin \gamma, \lambda_{4}=\cos \gamma-i \sin \gamma=1 / \lambda_{3}$; then $\xi=2(\cos \gamma-1)$, or $-4 \leqq \xi \leqq 0$. Thus, to satisfy the stability requirement $\xi$ must lie between 0 and -4 , and consequently can never be positive.

5. Numerical Results. A number of solutions were carried out with the anchor end of the line fixed and with the surface end forced to follow the motion of trochoidal waves of varying amplitudes and periods. Several typical solutions are reproduced here for the information of the reader. In Figures 3 and 4, plots are given of the maximum tension attained along the cable as a function of time for wave heights of 6 feet and periods of 12.5 seconds and 5 seconds, respectively. The periods of the variation in maximum tension correspond to the periods of the forced vibration, as expected. The maximum tension, however, increases in magnitude from 33,250 lbs in the case of waves of $12.5 \mathrm{sec}$ period to $49,500 \mathrm{lbs}$ when the period is 5 seconds. In Figure 5, the maximum tension attained for wave heights of $9 \mathrm{ft}$ and a period of 7.5 seconds is plotted. The maximum tension is approximately $60,000 \mathrm{lbs}$ as compared with 38,500 lbs for the case of 6 - $\mathrm{ft}$ waves with the same period.

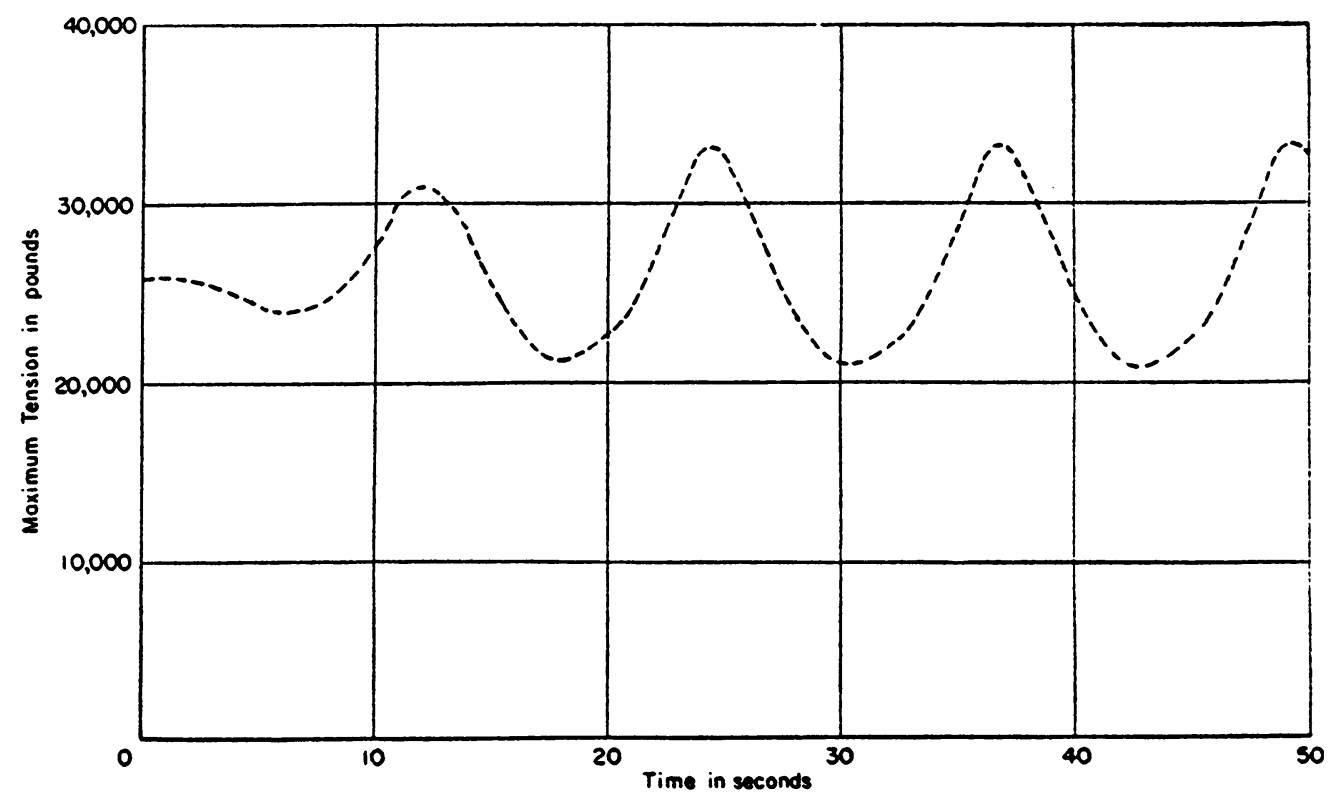

Fig. 3.-Mooring line oscillations: wave height, 6 feet; period, 12.5 seconds. 


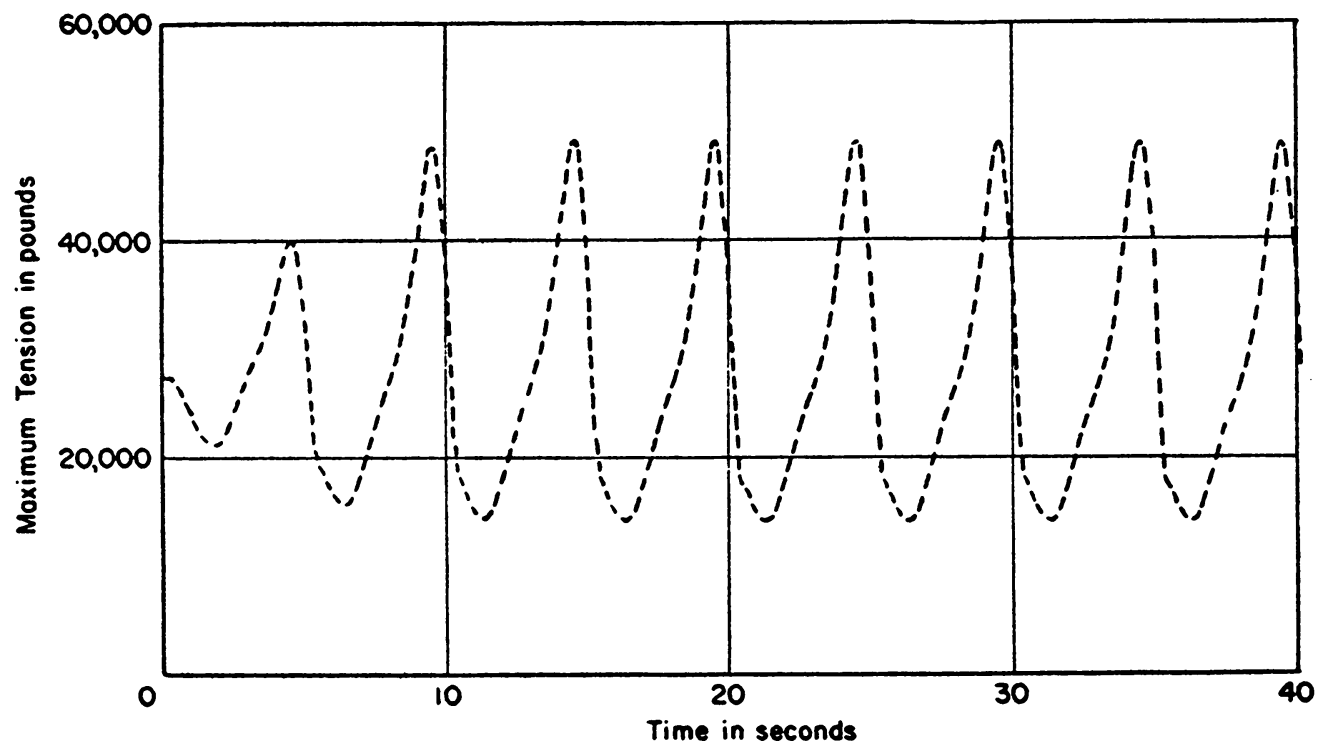

FIG. 4.-Mooring line oscillations: wave height, 6 feet; period, 5 seconds.

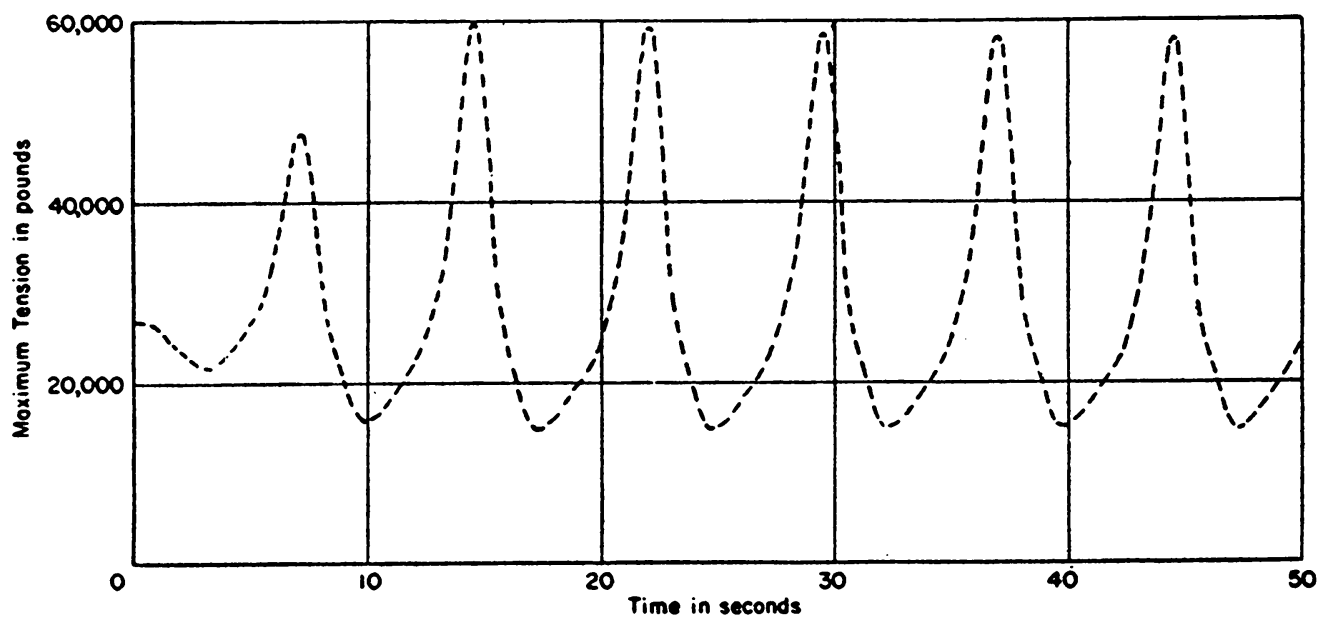

Fig. 5.-Mouring line oscillations: wave height, 9 feet; period, 7.5 seconds

As an experiment to aid in understanding the effect of the drag on the motion of the cable, one case was carried out with zero drag (i.e., motion in vacuum). A very interesting motion pattern was obtained which does not possess a periodic character. The resulting maximum tension is plotted in Figure 6.

The programming of the various phases of this problem was carried out by Mr. Thomas McFee, of the Applied Mathematics Laboratory, in a most effective manner. The speed and accuracy with which he accomplished this phase of the solution were largely responsible for the success in meeting the required time schedules. The authors would also like to express their gratitude to $\mathrm{Mr}$. R. T. McGoldrick, of the Structural Mechanics Laboratory, for proposing this problem and for a number of helpful discussions; to Dr. R. Bart, Structural Mechanics 


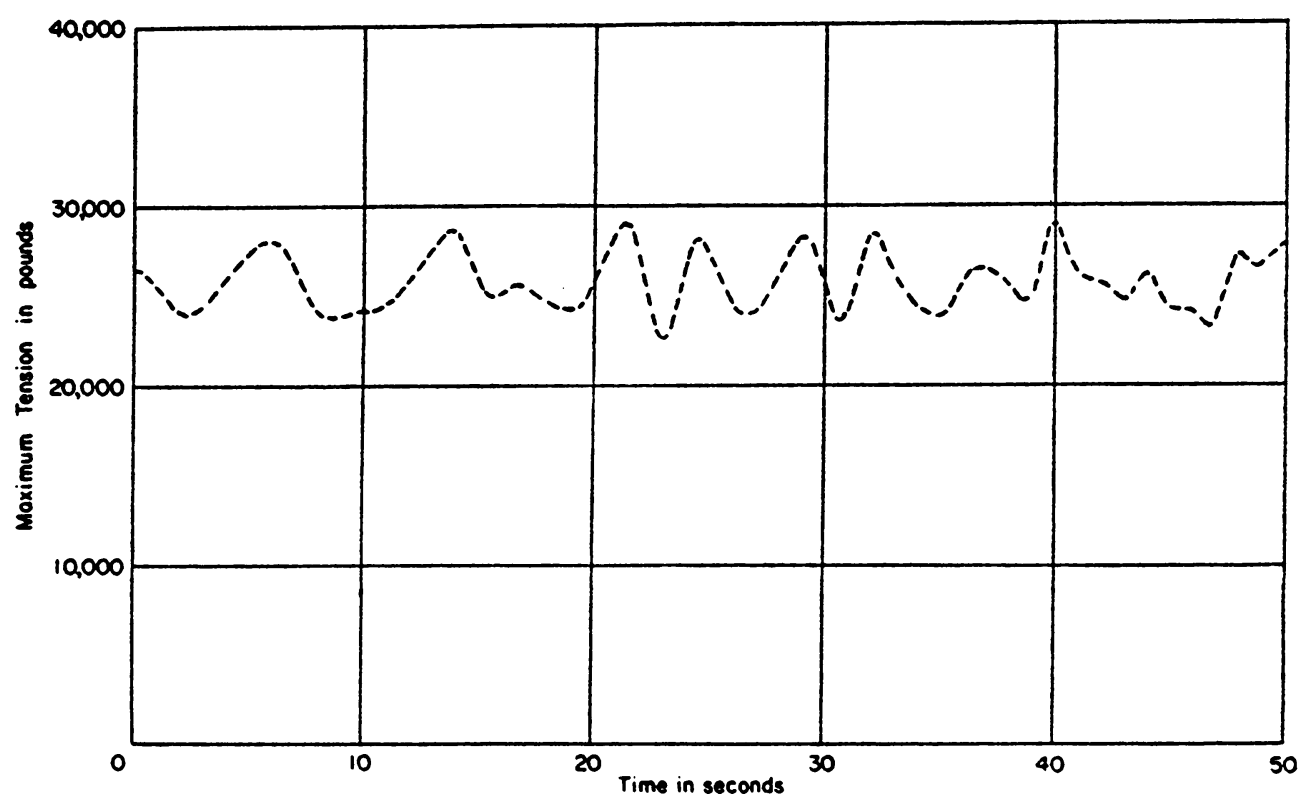

Fig. 6.-Mooring line oscillations without drag: wave height, 6 feet; period, 7.5 seconds.

Laboratory, for a number of ideas used in setting up the numerical procedure; to Dr. E. H. Kennard, David Taylor Model Basin, and Dr. R. M. Langer, Bureau of Ships, for helpful discussions in connection with the definition of the problem; to Dr. Daniel Shanks, Applied Mathematics Laboratory, for valuable suggestions; and to Miss Corinne Lundgren, Applied Mathematics Laboratory, for assistance in the preparation of the figures.

\section{Appendix - Notation.}

$C_{j+\frac{1}{3}}^{D}$ Drag coefficient for segment of cable between stations $j$ and $j+1$

$C_{j}^{x} \quad$ Resistance coefficient for horizontal motion of suspended prism

$C_{j}{ }^{\gamma} \quad$ Resistance coefficient for vertical motion of suspended prism

c Velocity of uniform horizontal current

$D$ Drag

$d_{j+1} \quad$ Diameter of segment of cable between stations $j$ and $j+1$

$e_{j+l} \quad$ Virtual mass of entrained fluid between stations $j$ and $j+1$

F Resultant force

$f_{i+1}^{D} \quad$ Drag factor for cable $=(\rho / 2) C_{j+l}^{D} l_{j+j} d_{j+1}$

$f_{j}^{X} \quad$ Horizontal drag factor for suspended prism $=(\rho / 2) C_{j}{ }^{X} S_{j}{ }^{X}$

$f_{j}{ }^{Y} \quad$ Vertical drag factor for suspended prism $=(\rho / 2) C_{j}{ }^{Y} S_{j}{ }^{Y}$

$g \quad$ Acceleration due to gravity

$I_{j} \quad$ Component of inertia tensor

$i \quad$ Imaginary unit

$J_{j} \quad$ Component of inertia tensor

$j \quad$ Subscript denoting station number along line

$K_{j} \quad$ Component of inertia tensor

$k_{j+1}$ Virtual inertia coefficient for segment of cable between stations $j$ and $j+1$ 
$l_{j+1} \quad$ Length of line between stations $j$ and $j+1$

$m_{j} \quad$ Mean mass of segments of cable adjoining station $j$

$m_{j}{ }^{*} \quad$ Mass of prism suspended from station $j$

$m_{j}{ }^{x} \quad$ Effective horizontal mass of suspended prism

$m_{j}{ }^{\gamma} \quad$ Effective vertical mass of suspended prism

$n \quad$ Superscript denoting time-step number

o Superscript denoting initial state (origin in time), or subscript denoting anchor end of line

$p$ Tangential component of velocity of cable (relative to medium)

$q$ Normal component of velocity of cable (relative to medium)

$S_{j}{ }^{x} \quad$ Projected area of suspended prism along $x$-axis

$S_{j}{ }^{\gamma} \quad$ Projected area of suspended prism along $y$-axis

8. Subscript denoting surface end of line

$T$ Tension

$t \quad$ Time

$\Delta t \quad$ Time-step interval

$u \quad$ Magnitude of velocity of cable (relative to medium)

$V_{j}^{*} \quad$ Volume of prism suspended from station $j$

$V_{j}^{x} \quad$ Equivalent volume of horizontal virtual mass of suspended prism

$V_{j}^{\gamma} \quad$ Equivalent volume of vertical virtual mass of suspended prism

$W_{j} \quad$ Mean net weight of segments of cable adjoining station $j$

$W_{j}{ }^{*} \quad$ Net weight of prism suspended from station $j$

$X \quad$ Horizontal component of resultant external force

$X_{j}^{*} \quad$ Horizontal component of damping force on suspended prism

$x \quad$ Horizontal coordinate of cable

$Y \quad$ Vertical component of resultant external force

$Y_{j}^{*} \quad$ Vertical ('omponent of damping force on suspended prism

$y \quad$ Vertical courdinate of cable

$\alpha \quad$ Damping coefficient of the perturbation functions

$\beta \quad$ Angular wave number of the perturbation functions

$\delta \quad$ The variation of

$\theta \quad$ Angle between horizontal and tangent to cable

$\lambda \quad$ Eigenvalue (root of characteristic equation)

$\mu_{j+1} \quad$ Linear density of segment of cable between stations $j$ and $j+1$

$\rho$ Density of fluid medium

$\sigma_{j+1}$ Cross-section area of segment of cable between stations $j$ and $j+1$

- Dot signifies differentiation with respect to time

$\sim$ Tilde signifies tentative value of a variable

Applied Mathematics Laboratory

David Taylor Model Basin

Washington 7, District of Columbia.

1. T. S. Walton \& H. Polachez, "Calculation of nonlinear transient motion of cables," David Taylor Model Basin Report 1279, 1959.

2. G. G. O'Brien, M. A. H YMan \& $\$$. Kaplan, "A study of the numerical solution of partial differential equations," Jn. Ilath. and Phys., v. 29, 1951, p. 223-251.

3. P. D. LAX \& R. D. RIChTMEYER, "Stability of difference equations," Com. Pure Appl. Math., v. 9, 1956, p. 267-293.

4. G. LUDFORD H. POLACHEx \& R. J. SEEGER, "On unsteady flow of compressible viscous fluids,"Jn. A ppl. Phys., v. 24, 1953, p. 490-495. 